\title{
Misdiagnosed infantile rhabdomyofibrosarcoma: A case report
}

\author{
TAO PAN $^{1 *}$, KEN CHEN $^{1 *}$, RUN-SONG JIANG ${ }^{2}$ and ZHENG-YAN ZHAO ${ }^{2}$ \\ Departments of ${ }^{1}$ General Surgery and ${ }^{2}$ Reconstructive Plastic Surgery, \\ The Children's Hospital of Zhejiang University School of Medicine, Hangzhou, Zhejiang 310003, P.R. China
}

Received January 19, 2015; Accepted February 16, 2016

DOI: $10.3892 / \mathrm{ol} .2016 .5032$

\begin{abstract}
Infantile rhabdomyofibrosarcoma is a rare form of soft-tissue tumor often associated with difficulties in diagnosis. The disease is positioned intermediately between rhabdomyosarcoma and infantile fibrosarcoma in terms of clinical presentation, immunohistochemistry, behavior, morphology and ultrastructural features. Reports of rhabdomyofibrosarcoma cases are limited in the literature. The present case describes a 26-month-old female who presented with a slowly progressive, soft-tissue mass in the right chest wall. The mass was successfully treated with surgery. Using histopathology, the tumor was diagnosed and classified as infantile rhabdomyofibrosarcoma. The patient has been followed-up for 2 years and is currently in good condition. The present case demonstrates that early, radical, local surgery and multidisciplinary cooperation were successful for the treatment of rhabdomyofibrosarcoma, and close follow-up highly recommended.
\end{abstract}

\section{Introduction}

Spindle cell tumors are a rare form of neoplasm that are often difficult to diagnose, with benign lesions occasionally exhibiting malignant and infiltrative features (1). Infantile rhabdomyofibrosarcoma is positioned intermediately between truculent spindle cell rhabdomyosarcoma and indolent, infantile fibrosarcoma in terms of clinical presentation, immunohistochemistry, behavior, morphology and ultrastructural features (2). It was first reported in 1993 (3). Infantile rhabdomyofibrosarcoma exhibits a few round rhabdomyoblastic cells with abundant cytoplasmic eosinophilia that are scattered within a uniform proliferation of spindle-shaped cells in a fasciculated pattern. By contrast,

Correspondence to: Dr Run-Song Jiang or Professor Zheng-Yan Zhao, Department of Reconstructive Plastic Surgery, The Children's Hospital of Zhejiang University School of Medicine, 57 Zhugan Lane, Yanan Road, Hangzhou, Zhejiang 310003, P.R. China

E-mail: jiangrs97@gmail.com

E-mail: zhaozy@zju.edu.cn

*Contributed equally

Key words: infantile fibrosarcoma, spindle cell rhabdomyosarcoma, infantile rhabdomyofibrosarcoma infantile fibrosarcoma has uniform, solidly-packed spindle cells arranged in a fascicular or herringbone pattern similar to the vascular pattern observed with hemangiopericytoma (4). Infantile rhabdomyofibrosarcoma may be identified as childhood spindle-cell sarcoma with a low degree of rhabdoid differentiation and aggressive clinical behavior (5). Overlooking the diagnosis of infantile rhabdomyofibrosarcoma may increase the chances of local recurrence or metastatic disease; therefore, aggressive multimodality treatment is required for these patients (6). Reports of rhabdomyofibrosarcoma cases are limited in the literature. The present case report describes a 26-month-old patient who presented with a slowly progressive, soft-tissue mass in the right chest wall, which was successfully resected.

\section{Case report}

A 26-month-old female was admitted to the Department of Plastic and Reconstructive Surgery at The Children's Hospital of Zhejiang University School of Medicine (Hangzhou, China) in October 2012 for the management of a large neoplasm in the right chest wall. The patient had been born at 38 weeks, weighing $3 \mathrm{~kg}$, following an uncomplicated pregnancy and delivery. The lesion had been present since birth and was $\sim 35 \times 80 \mathrm{~mm}$ in size. Over the previous 2 years, the mass had been painless and had slowly increased in size until 1 month prior to admission. No audible bruit, fluctuation or thrill was present, and the skin was normal on examination. Further physical examination was unremarkable, with the exception of a $6-\mathrm{cm}$ soft-tissue mass present in the right chest wall. Laboratory analysis indicated the following results: White blood cell count, $6.96 \times 10^{9}$ cells/ 1

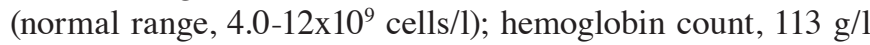
(normal range, 110-140 g/1); platelet count, 256x10 ${ }^{9}$ cells/1 (normal range, 100-400 $\times 10^{9}$ cells/1); and fibrinogen level, $2.15 \mathrm{~g} / 1$ (normal range, 1-5 g/l). Doppler ultrasound (iU Elite; Philips Healthcare, Amsterdam, The Netherlands) identified fluid collection in the subcutaneous tissue and muscle planes of the right chest wall. The tumor was initially suspected to be a lymphatic hemangioma. A posterior-anterior view of the chest was obtained using X-ray, which demonstrated that each lung field was expanded. There was no evidence of a lung mass or infiltrate, and the bilateral apices, bilateral pleural spaces and cardiac contour were all normal. The mediastinum and bilateral hila were also normal. A semicircle shadow with uniform density was observed to be protruding outwards from the soft tissue of 
the right chest wall. The lesion was $\sim 33 \times 77 \mathrm{~mm}$ in size, and no marked sorption or destruction of the ribs were detected (Fig. 1). The patient underwent a wide, local excision of the tumor, but did not undergo any chemotherapy following the surgical procedure. The surgical specimen was fixed with $10 \%$ neutral formaldehyde for $>24$ hour and paraffin embeded. The tissue slices (thickness, $4 \mu \mathrm{m}$ ) were stained with hematoxylin and eosin. Histopathology of the resected tumor identified that the spindle cells were arranged in fascicles with a herringbone pattern, and exhibited pale, eosinophilic cytoplasm and mitoses. A few round rhabdomyoblastic cells with abundant cytoplasmic eosinophilia that were scattered amoung uniform spindle-shaped cells, which were in a fasciculated pattern. (Fig. 2). However, no marked cross striation was observed in the rhabdomyoblastic cells. Immunohistochemical staining demonstrated strong positivity for vimentin, smooth muscle actin and desmin, and negativity for myogenin D1, S-100 and cluster of differentiation 34. According to the histopathological and immunohistochemical evidence the tumor was diagnosed as infantile rhabdomyofibrosarcoma. A computed tomography scan (SOMATOM Emotion 16; Siemens Healthcare, Erlangen, Germany) performed post-surgery did not reveal any signs of recurrence. Despite the aggressive nature of the lesion, there has been no evidence of recurrence or metastasis after 2 years of follow-up. Currently, the patient is alive and well. Written informed consent was obtained from the family of the patient for the publication of the present study.

\section{Discussion}

Infantile rhabdomyofibrosarcoma occupies an intermediate position between infantile fibrosarcoma and rhabdomyosarcoma in its clinical presentation, behavior morphology, immunohistochemical characteristics and ultrastructural features. Lundgren et al (3) described the first three cases of infantile rhabdomyofibrosarcoma, which were initially diagnosed as infantile fibrosarcoma. Two of the three patients with metastatic spread succumbed to the disease within 2 years of the primary operation, whereas the third patient is currently alive with a local recurrence. The three tumors deviated from infantile fibrosarcoma due to their clinical, ultrastructural, immunocytochemical and cytogenetic characteristics. An extensive comparative study was performed on these cases along with other cases of infantile fibrosarcoma (3).

Chaudhary et al (6) reviewed the limited number of infantile rhabdomyofibrosarcoma cases that were available in the literature. The median age of patients was 24 months (range, 13-48 months), with a male to female ratio of 5:2 with no specific predilection (6). In the study by Chaudhary et al, the cells were immunohistochemically positive for vimentin, smooth muscle actin and desmin, and negative for myoblobin. The present case demonstrated similarities with these previously reported studies, with the exception of the type of treatment undertaken. Myogenin has been reported to be highly specific and considered the most reliable marker for myogenesis, and Kumar et al (7) has hypothesized that myogenin is $100 \%$ specific for rhabdomyosarcoma. In addition, myogenin D1 is positive in the majority of rhabdomyoblastic cells and certain spindle cells (5). By contrast, myogenin and myogenin D1 are not expressed in infantile fibrosarcoma (8). Therefore, if a spindle-cell lesion has a high degree of muscle differentiation cross-striations and nuclear staining

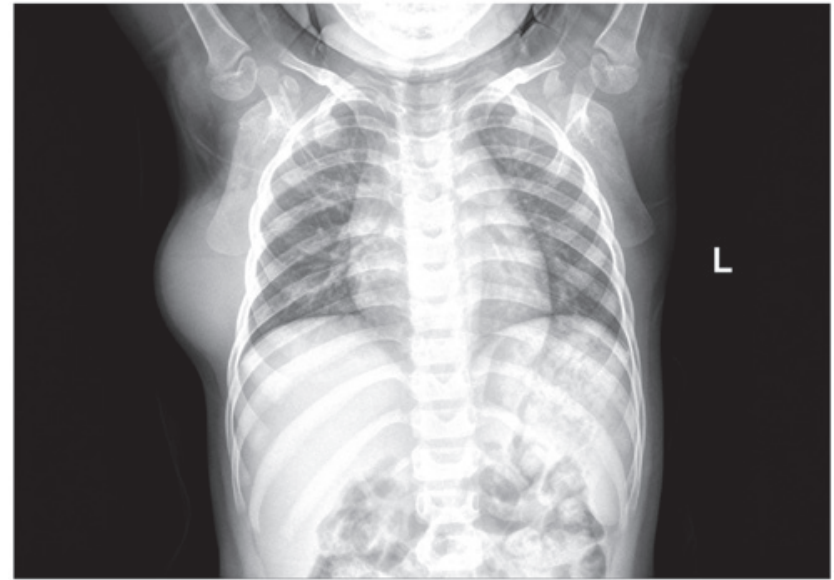

Figure 1. Posterior-anterior view of the chest using X-ray. The image shows a semicircle shadow with uniform density. L, left.

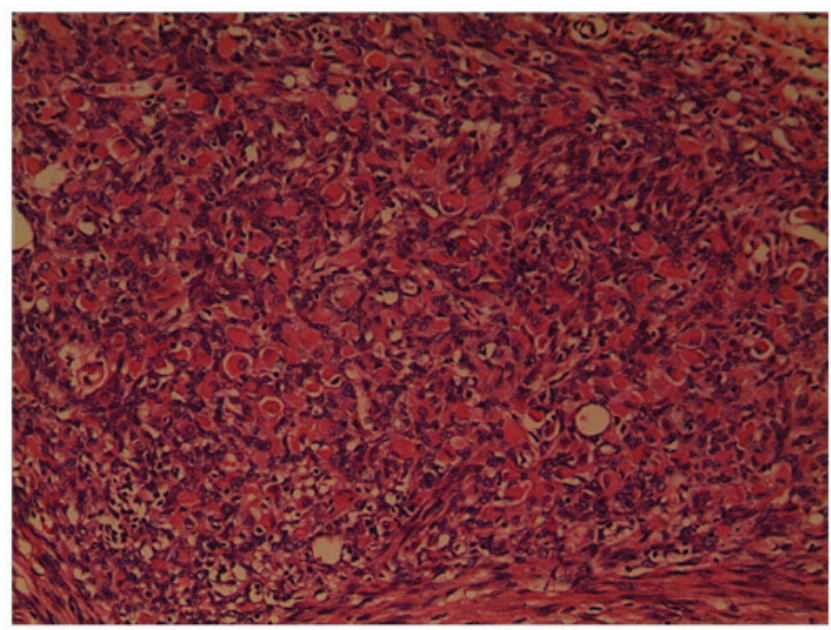

Figure 2. The resected tumor exhibited a few rhabdomyoblastic cells with abundant cytoplasmic eosinophilia, which were scattered within uniform spindle-shaped cells in a fasciculated pattern. Hematoxylin and eosin staining; magnification, x100.

for myogenin, the tumor would best diagnosed and classified as infantile rhabdomyofibrosarcoma.

Generally, aggressive multimodality treatment, including surgery and adjuvant chemotherapy, is required for the treatment of rhabdomyofibrosarcoma. However, in the present case, the patient was successfully treated with wide local excision alone, and presented no signs of recurrence after 2 years of follow-up. Although the patient was recommended to undergo adjuvant chemotherapy, her parents initially refused, but eventually agreed to chemotherapy with follow-up. Another case of infantile rhabdomyofibrosarcoma was treated at The Children's Hospital of Zhejiang University School of Medicine, in which an 8-month-old male patient presented with a tumor in the back. The patient underwent surgery with no follow-up chemotherapy. However, the neoplasm recurred at the same site 3 months later. In consequence, the patient underwent a second surgery, with immunohistochemical examination of the mass displaying similar features to the first tumor. The patient underwent one cycle of chemotherapy, which consisted of an intravenous drip of vindesine $\left(3 \mathrm{mg} / \mathrm{m}^{2}\right)$ and ifosfamide $\left(1300 \mathrm{mg} / \mathrm{m}^{2}\right)$ on the $1 \mathrm{st}$ 
day, then etoposide $\left(100 \mathrm{mg} / \mathrm{m}^{2}\right)$ on days $1-3$. The patient was followed-up for 3 years and had a good response to treatment (9).

In the present case, early and aggressive local surgery to remove the tumor, in addition to multidisciplinary cooperation, were successful in treating the disease. Close follow-up is highly recommended for a good prognosis in cases of infantile rhabdomyofibrosarcoma. Since the number of cases of infantile rhabdomyofibrosarcoma is extremely limited, additional accumulation of case data and extensive comparative analysis are required.

\section{Acknowledgements}

The authors would like to thank Mr. John Evans for reviewing the original manuscript (Zhejiang Ivy International High School, Hangzhou, China).

\section{References}

1. Gupta A, Maddalozzo J, Win Htin T, Shah A and Chou PM: Spindle cell rhabdomyosarcoma of the tongue in an infant: A case report with emphasis on differential diagnosis of childhood spindle cell lesions. Pathol Res Pract 200: 537-543, 2004.
2. Rao SI, Uppin SG, Ratnakar KS, Sundaram C and Senthil RP: Infantile rhabdomyofibrosarcoma: A distinct variant or a missinglink between fibrosarcoma and rhabdomyosarcoma? Indian J Cancer 43: 39-42, 2006

3. Lundgren L, Angervall L, Stenman G and Kindblom LG: Infantile rhabdomyofibrosarcoma: A high-grade sarcoma distinguishable from infantile fibrosarcoma and rhabdomyosarcoma. Hum Pathol 24: 785-795, 1993.

4. Weiss SW and Goldglum JR: Rhabdomyosarcoma. In: Soft Tissue Tumors. Enzinger FM and Weiss SW (eds). 4th edition. 1st Vol. Mosby Inc., Maryland Heights, MO, pp785-835, 2001.

5. Miki H, Kobayashi S, Kushida Y, Sasaki M, Haba R, Hirakawa E, Ogura K and Ohmori M: A case of infantile rhabdomyofibrosarcoma with immunohistochemical, electronmicroscopical, and genetic analyses. Hum Pathol 30: 1519-1522, 1999.

6. Chaudhary N, Shet T and Borker A: Infantile rhabdomyofibrosarcoma: A potentially underdiagnosed aggressive tumor. Int J Appl Basic Med Res 3: 66-68, 2013.

7. Kumar S, Perlman S, Harris CA, Raffeld M and Tsokos M: Myogenin is a specific marker for rhabdomyosarcomas: An imunohistochemical study in paraffin-embedded tissues. Mod Pathol 13: 988-993, 2000

8. Tang HF, Wang TL, Gu WZ, Lin L and Li MJ: Infantile rhabdomyofibrosarcoma. Zhonghua Bing Li Xue Za Zhi 34: 607-608, 2005 (In Chinese).

9. Cessna MH, Zhou H, Perkins SL, Tripp SR, Layfield L, Daines C and Coffin CM: Are myogenin and myo-D1 expression specific for rhabdomyosarcoma? A study of 150 cases with emphasis on spindle cell mimics. Am J Surg Pathol 25: 1150-1157, 2001. 